\title{
Retraction Note to: Access Requirement Analysis of E-Governance Systems
}

\author{
Tai-hoon Kim \\ Multimedia Engineering Department, Hannam University, \\ Daejeon, Korea \\ taihoonnahnu.kr
}

T.-h. Kim, A. Stoica, and R.-S. Chang (Eds.): SUComS 2010, CCIS 78, pp. 124-130, 2010.

(C) Springer-Verlag Berlin Heidelberg 2010

DOI 10.1007/978-3-642-16444-6_82

The paper starting on page 124 of this volume has been retracted because it is a duplicate of the paper starting on page 466 of the same volume. 